\title{
Nevi presenting a halo: Sutton nevus, Meyerson nevus, and Wollina-Schaarschmidt halo-like dermatosis
}

\author{
Uwe Wollina
}

Department of Dermatology and Allergology, Hospital Dresden-Friedrichstadt, Academic Teaching Hospital of the Technical University of Dresden, Friedrichstrasse 41, 01067 Dresden, Germany

Corresponding author: Prof. Uwe Wollina, E-mail: wollina-uw@khdf.de

\begin{abstract}
Melanocytic nevi uncommonly develop halo-like phenomena. In this short review, we will discuss the major three types. Sutton nevus - more common in vitiligo - is characterized by loss of pigmentation around moles. Mimics of Sutton nevus have occasionally been reported in melanoma, although Sutton nevus itself is benign. Meyerson nevus is a pruritic lesion with a central mole surrounded by an eczematous halo. It is not necessarily associated with atopic dermatitis. The Wollina-Schaarschmidt phenomenon describes the halo-like skin free of psoriasis in patients with psoriasis.
\end{abstract}

Key words: Melanocytic nevi; Sutton nevus; Meyerson nevus; Wollina-Schaarschmidt phenomenon; Psoriasis; Melanoma

\section{INTRODUCTION}

Melanocytic nevi may undergo various changes of the clinical presentation, e.g. shape, thickness, size, color, texture. Some nevi develop halo-like changes surrounding the primary pigmented lesion. Here we will discuss three types of melanocytic nevi with a halo.

\section{Sutton Nevus}

The most common and well known is nevus Sutton or halo nevus first described in medical journals in 1916, where a hypopigmented area develops around a pre-existing melanocytic nevus. Halo nevi undergo specific sequelae: In Stage I the nevus remains brown, in stage II its pigment can disappear leading to a pink-colored papule. In stage III the central papule disappears, leading to a circular area of depigmentation. Eventually, in stage IV the depigmented area may show repigmentation [1]. The loss of color is the result of a cytotoxic T-cell attack against melanocytes and can lead to a complete disappearance of the mole [2]. Halo nevi are more common in vitiligo. About one quarter of children with vitiligo also have halo nevi [3].

A similar phenomenon has been observed in melanoma during treatment with check point inhibitors [4].

\section{Meyerson Nevus}

Another phenomenon is the Meyerson nevus, a pruritic halo-like dermatitis around moles. The skin shows lichenification, sometimes redness or scaling (Figs. 1 and 2). In the initial description of 1971, the suspected cause was pityriasis rosea but the concept has been changed nowadays to an eczematous reaction of unknown cause. Meyerson nevus is caused by an inflammatory infiltrate surrounding a preexistent mole predominated by CD4+ T-lymphocytes, that miss other markers of $\mathrm{T}$ helper cells such as BCL-6 or CD10. This peculiar nevus type is symptomatic by pruritic sensations. Histopathological evaluation reveals a spongiotic dermatitis resembling eczema [2-5]. 


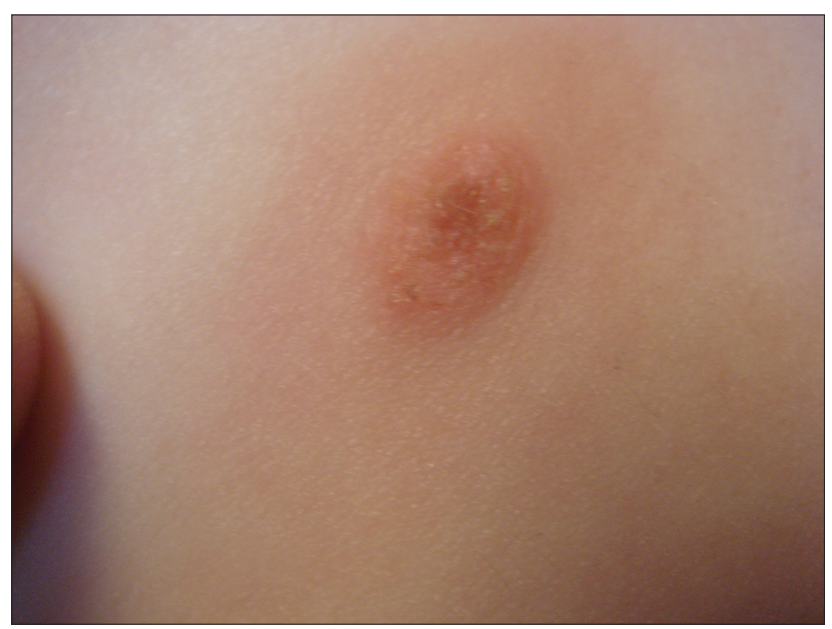

Figure 1: Typical Meyerson nevus with lichenoid pruritic halo in a girl of 15 months.

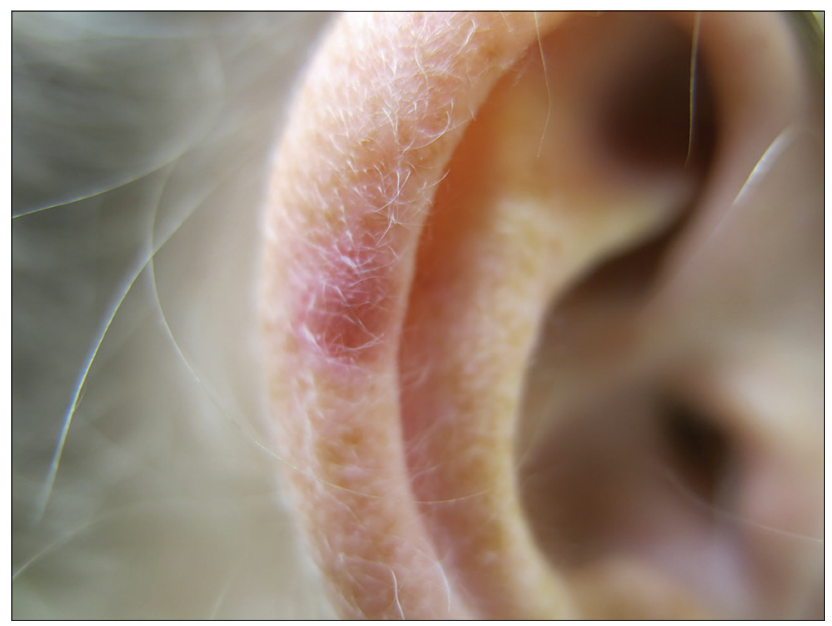

Figure 2: Clinical atypical Meyerson nevus with redness on the ear helix in a 27-year-old woman. Most of her moles were red.

Meyerson nevus in children and adolescents is rarely reported in the medical literature although it can be observed from time to time in the outpatient clinic. It may spontaneously regress and is not dependent on atopic disposition. Symptomatic relief is easily achieved by topical corticosteroids, recurrence after cessation of treatment is uncommon [6,7]. We would recommend treatment since scratching may lead to morphologic changes in the nevus component and unnecessary surgery.

In adults, Meyerson nevus is uncommon. A pruritic melanocytic lesion can be suspicious for melanoma, although Meyerson phenomenon has only rarely been observed with early melanomas [8,9]. Dermoscopy may be used for a more detailed evaluation. In any case suspicious for malignancy, complete excision of the lesion including the halo, is recommended in adults [10].

\section{Wollina-Schaarschmidt Phenomenon}

Another halo-like phenomenon had been observed among patients with psoriasis - the WollinaSchaarschmidt phenomenon described in 1990. In a study on 76 psoriasis patients, we found melanocytic nevi within psoriatic lesions in $7 \%$, and in perilesional areas of about $2 \mathrm{~cm}$ in $13 \%$ of the patients. Under magnification (operating microscope), we failed to detect any signs of psoriasis in both the epidermis covering the nevi and the adjacent epidermis. Histopathologically, the adjacent epidermis was free of psoriasis. Using unfixed frozen sections in histochemistry, we studied the lectin binding of FITClabeled ConA and UEA I in 5 nevi. The epidermal reaction was comparable to that of psoriatic lesions. In contrast to psoriatic lesions, there was no staining of the spinous layer with a polyclonal antiserum against calmodulin. We suggested possible 'protective' factors of melanocytic nevi against psoriasis $[11,12]$.

\section{CONCLUSIONS}

Halo-like features may occur around benign melanocytic nevi. They have different etiologies. Dermatologists should be familiar with these lesions and their melanoma mimics.

\section{REFERENCES}

1. Sotiriadis D, Lazaridou E, Patsatsi A, Kastanis A, Trigoni A, Devliotou-Panagiotidou D. Does halo nevus without halo exist? J Eur Acad Dermatol Venereol. 2006;20:1394-6.

2. Fernandez-Flores A, Saeb-Lima M. The inflammatory infiltrate of melanocytic nevus. Rom J Morphol Embryol. 2014;55:1277-85.

3. Cohen BE, Mu EW, Orlow SJ. Comparison of childhood vitiligo presenting with or without associated halo nevi. Pediatr Dermatol. 2016;33:44-8.

4. Harvey NT, Millward M, Macgregor K, Bucat RP, Wood BA. Cutaneous metastatic melanoma resembling a halo nevus, in the setting of PD-1 inhibition. Am J Dermatopathol. 2016;38:e159-62.

5. Meyerson LB. A peculiar papulosquamous eruption involving pigmented nevi. Arch Dermatol. 1971;103:510-12.

6. Rolland S, Kokta V, Marcoux D. Meyerson phenomenon in children: observation in five cases of congenital melanocytic nevi. Pediatr Dermatol. 2009;26:292-7.

7. Schepis C, Siragusa M. The Meyerson phenomenon in a teenager. Dermatol Online J. 2008;14:28.

8. Sezer E, Özturk Durmaz E, Çetin E, Şahin S. Meyerson phenomenon as a component of melanoma in situ. Acta Dermatovenerol Croat. 2016;24:81-2.

9. Rodins K, Byrom L, Muir J. Early melanoma with halo eczema (Meyerson's phenomenon). Australas J Dermatol. 2011;52:70-3.

10. Ferneiny M, Pansé I, Schartz N, Battistella M, Verola O, Morel P, et al. [Disseminated perinaevic Meyerson phenomenon revealing melanoma] (French). Ann Dermatol Venereol. 2012;139:137-41. 
www.odermatol.com

11. Wollina U, Schaarschmidt H, Henkel U, Roth H, Barta U, Hipler C. [A combination of psoriasis vulgaris and nevus cell nevus--clinical, histopathologic and histochemical findings] (German). Z Hautkr. 1990;65:381-2,385-7.

12. Wollina U. Calmodulin in human skin--localizations and interpretations. Arch Dermatol Res. 1993;285:310-1.
Copyright by Uwe Wollina. This is an open-access article distributed under the terms of the Creative Commons Attribution License, which permits unrestricted use, distribution, and reproduction in any medium, provided the original author and source are credited.

Source of Support: Nil, Conflict of Interest: None declared. 